\title{
What Clinical Interventions Have Been Implemented to Prevent or Reduce Postpartum Hypertension Readmissions? A Clin-IQ
}

Sara O'Meara

Molly Lepic

Follow this and additional works at: https://aah.org/jpcrr

Part of the Family Medicine Commons, and the Obstetrics and Gynecology Commons

\section{Recommended Citation}

O'Meara S, Lepic M. What clinical interventions have been implemented to prevent or reduce postpartum hypertension readmissions? A Clin-IQ. J Patient Cent Res Rev. 2016;3:150-2. doi: 10.17294/

2330-0698.1264

Published quarterly by Midwest-based health system Advocate Aurora Health and indexed in PubMed Central, the Journal of Patient-Centered Research and Reviews (JPCRR) is an open access, peer-reviewed medical journal focused on disseminating scholarly works devoted to improving patient-centered care practices, health outcomes, and the patient experience. 


\title{
What Clinical Interventions Have Been Implemented to Prevent or Reduce Postpartum Hypertension Readmissions? A Clin-IQ
}

\author{
Sara O'Meara, DO, Molly Lepic, DO \\ Department of Obstetrics and Gynecology, Aurora Sinai Medical Center, Aurora Health Care, Milwaukee, WI
}

\begin{abstract}
A literature review was conducted to determine what clinical interventions have been studied and implemented to prevent and/or reduce postpartum hypertension readmissions. Appropriate verbal and printed educational materials should be given to the patient prior to discharge with use of the "teach back" method. Patients and health care providers within the multidisciplinary team should be educated on the warning signs and symptoms of worsening hypertensive disease and when to appropriately involve the obstetrician. The use of text messaging may be useful in preventing hospital readmissions by increasing patient follow-up and compliance and appropriately managing patients in the postpartum period. Treating postpartum patients with furosemide may decrease blood pressure and prevent postpartum hypertension and the need for antihypertensive therapy. (J Patient Cent Res Rev. 2016;3:150-152.)
\end{abstract}

Keywords postpartum hypertension; readmission; prevention

\section{Clinical Question}

What clinical interventions have been implemented to prevent or reduce postpartum hypertension readmissions?

\section{Answer}

Limited studies have reported on clinical interventions focused on preventing and reducing postpartum hypertension readmissions. To date, no reliable evidence yet exists to appropriately guide management for these patients in the postpartum period, though emphasis has been placed on appropriate patient education, targeting at-risk patients and establishing proper follow-up after discharge.

Date answer was determined: March 2016.

Level of evidence: B.

Inclusion Criteria

Publications from January 2006 to March 2016

Correspondence: Sara M. O'Meara, DO, Aurora Sinai Medical Center, 945 N. 12th Street, Milwaukee, WI, 53233, T: 414-219-5800,

Email: sara.o'meara@aurora.org that were searchable in PubMed or Google Scholar, written in the English language, and that focused on clinical interventions to reduce/prevent postpartum hypertension readmissions.

\section{Exclusion Criteria}

Publications reporting readmissions for other causes, such as infection, headache without hypertension, venous thromboembolism or cardiac disease unrelated to hypertension, were excluded, as were editorials and perspectives. Publications providing antepartum hypertensive guidelines also were excluded, as these did not appropriately address the clinical question.

\section{Summary of Problem}

Hospital readmission rates are a critical component in health care performance measures and costs, and have been the focus of the Centers for Medicare and Medicaid Services' attention, thus prompting efforts to improve health care quality and patient education. ${ }^{1}$ In $2009,27 \%$ of obstetric readmissions were due to hypertensive disease. ${ }^{2}$ Maternal blood pressure peaks 3-6 days after delivery, when patients have already been discharged home. ${ }^{3}$ As a result, postpartum hypertension readmissions make up a significant portion of obstetrical readmissions and contribute to 
overall hospital readmission rates. Factors contributing to postpartum hypertension readmissions rates may include: provider and patient lack of knowledge regarding the disease process and progression, lack of standardized management and treatment plans, and insufficient patient education. Task Force on Hypertension in Pregnancy guidelines have been established by the American College of Obstetricians and Gynecologists for management of maternal hypertension in labor and delivery, but these do not address management during the postpartum period. ${ }^{1}$ Postpartum hypertension not only significantly impacts health care costs but also contributes to maternal morbidity including stroke and mortality. ${ }^{3}$

\section{Summary of Evidence}

Of the initial 58 studies identified by our search strategy, only five satisfied the inclusion and exclusion criteria. The first finding from our search was a presentation within the American Hospital Association's "Health Care Leader Action Guide to Reduce Avoidable Readmissions." "2 This summary report identified several strategies to reduce avoidable readmissions throughout patient care. During hospitalization, it is recommended that health care providers: screen patients for risk factors for readmission and tailor care appropriately, establish effective communication with the patient and family, use the "teach back" method for education and comprehension (i.e. patients are asked to reiterate their understanding of the information communicated), and coordinate patient care across multidisciplinary teams. Upon discharge, it is recommended that caregivers: implement comprehensive discharge planning, use the "teach back" method for discharge planning, and schedule follow-up appointments as indicated. Postdischarge, it is recommended that health care providers: promote patient self-management of their diagnosis, conduct home visits, and follow up with the patient via the telephone. ${ }^{2}$

Al-Safi et al. studied demographics and clinical factors that may play a role in delayed postpartum preeclampsia and eclampsia, thereby contributing to postpartum hypertension readmissions. ${ }^{4}$ In terms of the demographics collected - age, gravidity, body mass index, ethnicity, history of hypertensive disease and other comorbidities - young age was the only significant demographic variable for developing eclampsia. All patients studied presented with prodromal symptoms, with headache being the most common. The authors emphasized the need to educate emergency department providers on prodromal symptoms given that most of the patients were initially evaluated in this setting, and patients on warning signs and symptoms of their diagnosis prior to discharge to potentially avoid complications and development of postpartum preeclampsia or eclampsia. Patient education should occur prior to hospital discharge using both verbal and printed materials. ${ }^{4}$

With a design based on evidence that hypertensive complications frequently occur within 7 days postpartum, the third study evaluated the use of provider-patient text messaging as an avenue for increased outreach prior to follow-up appointments. ${ }^{5}$ The text messaging intervention sought "to provide reassurance, answers to questions and problem-solving solutions, and to identify women who needed escalated care." A member of the patient's health care team sent a standardized text message to patients within 3 to 4 days following hospital discharge. Response rate was $34 \%$, and $27 \%$ required a follow-up phone call for additional information and triage. Participants reported satisfaction with this method of enhanced communication and follow-up; however, further research needs to be performed to determine if this method reduces readmission rates. ${ }^{5}$

A Cochrane review titled "Prevention and Treatment of Postpartum Hypertension" sought to determine if any evidence-based recommendations exist to guide management (though not specifically to determine ways to reduce readmissions, as was our goal) of postpartum hypertensive patients. ${ }^{3}$ The review included nine trials, all of which studied the effects of different medications commonly used in pregnancy and in the postpartum period, including furosemide, nifedipine, timolol, hydralazine, methyldopa and labetalol. The review concluded that "postnatal furosemide may decrease the need for postnatal antihypertensive therapy in the hospital, but more data are needed on substantive outcomes before this practice can be recommended." 3 The authors suggested that the use of furosemide may be beneficial in reducing blood pressure, thus potentially reducing readmission rates and maternal health complications. 
Understanding the clinical factors associated with postpartum hypertension readmissions may assist in identifying and targeting appropriate patient populations. In a study conducted by Hirshberg et al., several labor, delivery and postpartum factors, such as demographics, mode of delivery, length of labor, severity of hypertensive disease, use of magnesium sulfate, fluid balance, initiation of antihypertensive medication and postpartum blood pressure prior to discharge, were examined to determine their association with hospital readmission. ${ }^{1}$ Interestingly, readmitted patients were noted to have longer lengths of labor and were less likely to be initiated on antihypertensive therapy prior to discharge.

\section{Conclusions}

Provider and patient education, verbal and printed resources, text messaging with prompt follow-up postdischarge and the use of postnatal furosemide appear to exhibit the potential to decrease the morbidity and mortality associated with postpartum hypertensive disease and hospital readmission.

\section{Patient-Friendly Recap}

- There are currently no evidence-based management guidelines for treating postpartum hypertension.

- The authors reviewed published reports for evidence that may support any clinical interventions to prevent hospital readmission caused by postpartum hypertension.

- They found that providing educational materials to both caregivers and patients, administering furosemide after the birth, and use of two-way text messaging for follow-up management all may serve to decrease related complications and readmissions.

\section{Acknowledgments}

This review was conducted as part of the Alliance of Independent Academic Medical Center's National Initiative $\mathrm{V}$ on reducing health disparities, with consultative support by Carla Kelly, DO, Jeffrey Stearns, MD, and Deborah Simpson, PhD, all of Aurora Health Care.

The Clin-IQ teaching materials used for this project were provided by Oklahoma Shared Clinical and Translational Resources, funded by a National Institutes of Health grant (NIGMS U54GM104938).

\section{Conflicts of Interest}

None.

\section{References}

1. Hirshberg A, Levine LD, Srinivas SK. Clinical factors associated with readmission for postpartum hypertension in women with pregnancy-related hypertension: a nested case control study. J Perinatol. 2016;36;405-9. CrossRef

2. Muri JH, Crawford N, Connors Jellen B; American Hospital Association. Reducing avoidable obstetrical and neonatal readmissions. http://www.aha.org/content/11/ PerinatalReadmissionscall1.pdf. Accessed Feb. 3, 2016.

3. Magee L, von Dadelszen P. Prevention and treatment of postpartum hypertension. Cochrane Database Syst Rev. 2013;(4):CD004351. CrossRef

4. Al-Safi Z, Imudia AN, Filetti LC, Hobson DT, Bahado-Singh RO, Awonuga AO. Delayed postpartum preeclampsia and eclampsia. Obstet Gynecol. 2011;118;1102-7. CrossRef

5. Scalise LF, Stringer M. Follow-up text messages for patients at high risk of postpartum hypertension. (abstr.) J Obstet Gynecol Neonatal Nurs. 2015;44:S6. CrossRef

(C) 2016 Aurora Health Care, Inc. 